\title{
Fatores Facilitadores do Desempenho Inovador e Desempenho Organizacional: Estudo na Indústria de Móveis de Madeira no Estado de Santa Catarina
}

\author{
Leonardo Rodrigues Thomaz Bridi ${ }^{1}$, Giancarlo Gomes², Pedro Lucas Resende Melo \\ ${ }^{1}$ Instituto Federal Catarinense - leonardo.bridi@ifc-videira.edu.br \\ ${ }^{2}$ Universidade Regional de Blumenau (FURB) - giancarlo@pzo.com.br \\ ${ }^{3}$ Universidade Paulista (UNIP) - resendemelo@usp.br
}

\section{PALAVRAS CHAVE}

Fontes de Inovação;

Orientação Empreendedora.

Desempenho Inovador;

Desempenho Organizacional;

Indústria de Móveis de Madeira.

Recebido 31.05.2017

Revisado 04.09.2017

Aceito 28.11.2017

ISSN 1980-4431

Double blind review

\section{RESUMO}

O presente estudo tem por objetivo analisar a influência das Fontes de Inovação e da Orientação Empreendedora no Desempenho Inovador e Desempenho Organizacional na indústria de móveis de madeira do estado de Santa Catarina. A pesquisa foi quantitativa, descritiva e causal, com método survey e com corte transversal. Os dados foram analisados por meio de Modelagem de Equações Estruturais com o auxílio do software Smart PLS. Os resultados do modelo proposto mostraram relação positiva entre Fontes de Inovação e da Orientação Empreendedora no Desempenho Inovador e consequentemente no Desempenho Organizacional. Por fim, os resultados do modelo proposto mostraram significância e confiabilidade sustentando que o desempenho inovador propiciará resultados positivos no Desempenho Organizacional, nomeadamente na fidelidade dos clientes, crescimento das vendas, rentabilidade e retorno sobre o investimento.

\section{Introdução}

Estudos ligados ao empreendedorismo têm atraído o interesse de pesquisadores (Ireland, Reutzel, \& Webb, 2007). O empreendedorismo abrange atos de criação organizacional e renovação, ocorrendo dentro ou fora de uma organização (Sharma \& Chrisman, 1999). Segundo Ireland e Webb (2005) a pesquisa em administração relacionada ao empreendedorismo tem focado na Orientação Empreendedora (OE), que procura explicar como o empreendedorismo é colocado em prática.

Estudo anteriores como Ireland e Webb (2005), Rauch et al. (2009) e Runyan, Droge e
Swinney (2008) indicam relação positiva entre OE e Desempenho Organizacional. Por outro lado, alguns resultados obtidos por George, Wood e Khan (2001) não foram totalmente conclusivos. Para Thoumrungroje e Tansuhaj (2005) isto pode ser devido ao fato que o conceito de Desempenho Organizacional pode ser influenciado tanto por variáveis internas quanto externas.

$\mathrm{O}$ aumento na capacidade de inovação das empresas do setor de móveis constitui uma condição importante para garantir a inserção competitiva tanto no mercado doméstico como no mercado externo segundo a Agência Brasileira de Desenvolvimento [ABDI] (2009). A partir desta premissa, a inovação aparece como um conceito

Revista de Negócios, v. 21, n. 2, p. 51-66, April, 2016. 
basilar para o crescimento do setor de móveis de madeira de Santa Catarina. Sendo assim, consequentemente, a inovação se transforma em um elemento que atua significativamente para conduzir o Desempenho Organizacional. Na mesma linha de raciocínio, os estudos sobre inovação e a sua vinculação com resultados de Desempenho Organizacional e consequente manutenção das empresas em longo prazo, têm sido abordadas e discutidas na literatura (Damanpour, 1991) (Tidd, Bessant \& Pavitt, 2005).

Ao considerar a influência da inovação no Desempenho Organizacional é relevante destacar as fontes de inovação. Freeman (1995) considerou que as fontes de inovação quando originárias em conhecimentos científicos, propiciam a introdução de inovações radicais, e por outro lado quando as tecnologias estão dominadas, aparecem condições para aparecimento de inovações incrementais.

Ireland, Reutzel e Webb (2007) sustentam que o empreendedorismo é um campo de estudos para o qual ainda é necessário progredir continuamente pela busca por uma teoria distinta. Isso se justifica pelo fato de existirem poucos estudos empíricos que contemplem o desenvolvimento do paradigma e também pela dispersão de enfoques existentes nestas pesquisas. Trata-se, portanto, de um campo fértil a ser explorado pelos pesquisadores na busca pela construção da teoria relacionada ao empreendedorismo.

Estima-se que as fontes de inovação e a orientação empreendedora são relevantes para uma melhor compreensão da relação entre 0 desempenho em inovação de produtos e processos entre OE e Desempenho Organizacional, sendo esses conceitos apresentados por Chiva e Alegre (2013) que acrescentam que segundo Nelson (1991) e Zott (2003) as fontes de inovação e a OE são consideradas antecedentes do desempenho em inovação.

Com base na revisão da literatura e nas concepções teóricas apresentadas, entende-se que as fontes de inovação e a orientação empreendedora são considerados facilitadores da inovação e consequentemente do desempenho organizacional. Destarte, diante do exposto o objetivo do estudo é analisar a influência das Fontes de Inovação e da Orientação Empreendedora no Desempenho Inovador $e$ Desempenho Organizacional de empresas da

\section{indústria de móveis de madeira de Santa Catarina?}

$\mathrm{O}$ artigo encontra-se estruturado em cinco seções. A primeira seção corresponde a introdução do artigo, sendo compreendida pela apresentação do tema de pesquisa e problema a ser trabalhado. A segunda seção trata do modelo teórico. Na terceira seção é estruturada o método da pesquisa. Posteriormente, na quarta seção têm-se a análise dos dados. Na quinta seção as considerações finais a partir dos resultados demonstrados pela presente pesquisa e, por fim, as referências bibliográficas.

\section{Revisão de literatura}

A revisão da literatura é composta pelos construtos que antecedem a inovação e o desempenho organizacional. As dimensões são apresentadas na sequência.

\subsection{Modelo Teórico Proposto}

Este modelo considera os constructos de Fontes de Inovação, OE, Desempenho Inovador (eficácia de inovação do produto e eficiência no processo de inovação) apresentado por FernándesMesa e Alegre (2015) e Desempenho Organizacional.

\subsubsection{Fontes de Inovação}

Pesquisas anteriores de Schoenmakers e Duysters (2006) e Tsai (2009) sugerem que uma empresa pode ampliar sua inovação de produtos por meio da interação com diferentes colaboradores, fornecedores, clientes, concorrentes e institutos de pesquisa. Fornecedores geralmente têm conhecimento maior e mais abrangente sobre as peças e componentes que podem ser críticos para o desenvolvimento de novos produtos.

Colaborar com os clientes é outra maneira importante para que uma empresa melhore o seu desempenho em inovação de produtos (Gupta et al., 2000). Trabalhar com clientes não só proporciona benefícios na identificação de oportunidades de mercado, mas também reduz a probabilidade de um projeto deficiente nos estágios iniciais do seu desenvolvimento (Tsai, 2009).

No que diz respeito aos concorrentes, o objetivo da cooperação com eles, em termos gerais, é realizar pesquisas básicas e estabelecer padrões. Assim, é provável que as empresas

Revista de Negócios, v. 21, n. 2, p. 51-66, April, 2016. 
cooperem com os concorrentes sempre que partilham problemas comuns que estão fora da área de influência do concorrente, tais como programas de pesquisa e acordos de coprodução. (Zeng, et al., 2010). Para Inkpen e Pien (2006) as empresas que colaboram com os concorrentes podem ter um melhor desempenho em inovação. Ainda, a colaboração com os concorrentes permite às empresas avaliarem o nível tecnológico dos seus concorrentes. As empresas que conhecem melhor as estratégias tecnológicas de seus concorrentes são mais capazes de se diferenciar.

Devido aos incentivos dos governos, as empresas estão buscando inovações de produtos, colaborando com universidades e instituições de pesquisa. As universidades e os institutos de pesquisa são centros importantes para a criação e divulgação de conhecimentos científicos (Hemmert, 2004). Institutos de pesquisa são uma fonte importante de novos conhecimentos científicos. Colaborar com organizações de pesquisa permite que uma empresa acesse conhecimento científico previamente inexplorado. Esse conhecimento pode fornecer à empresa diferentes modos de raciocínio e de resolução de problemas. Hemmert (2004) e Tsai (2009).

\subsubsection{Orientação Empreendedora}

Miller (1983) definiu a atividade empreendedora como um processo de comportamento empreendedor constituído por três capacidades: agir de forma inovadora (inovativeness), agir de forma proativa (proactiveness) e assumir riscos (risk-taking). Posteriormente, Lumpkin e Dess (1996) e procuraram compilar as contribuições teóricas e práticas no sentido de estabelecer uma ligação entre os processos empreendedores e o desempenho das organizações. Com o intuito de promover esse elo acrescentaram às três capacidades ou competências definidas por Miller (1983), mais duas: autonomia (autonomy) e competitividade agressiva (competitive agresssiveness).

Foi a partir destas cinco competências que Lumpkin e Dess (1996) caracterizaram a OE. Lumpkin e Dess (1996) sustentam que o construto da OE é multidimensional e independente. Nesse caso, pode ser considerada que uma organização possui $\mathrm{OE}$ mesmo que esta não tenha a totalidade das competências antes apresentadas, uma vez que o aumento ou diminuição da $\mathrm{OE}$ pode ser o resultado de alterações em todas ou algumas dessas competências.

A inovação, realizada dentro do escopo da OE, pode ser compreendida pelo seu caráter original e criativo e consequentemente na capacidade competitiva uma vez que fornece subsídios às novas ideias, experiências, geração de novos produtos, processos e tecnologias previamente existentes Lumpkin e Dess (1996) e Wiklund e Sheperd (2005).

Fernándes-Mesa e Alegre (2015) procurou observar de que forma a OE impacta no desempenho inovador e usou como variável de moderação o tamanho da indústria. Nesta pesquisa, os indicadores da OE que foram considerados são: Inovação/Inovatividade; Assumir riscos; e Proatividade e foram observadas pelo prisma multidimensional proposto por Lumpkin e Dess (1996).

\subsubsection{Desempenho Inovador}

Nesta pesquisa o desempenho inovador foi dividido nas dimensões: eficácia e eficiência. Essa perspectiva foi trabalhada inicialmente por Alegre, Lapiedra e Chiva (2006). Os autores desenvolveram uma escala de medida de desempenho em inovação de produtos denominado: Desempenho Inovador. Este constructo foi usado a partir da validação da escala em língua portuguesa realizada no estudo de Gomes (2013), tendo este, por sua vez, base na Organização para Cooperação e Desenvolvimento Econômico [OCDE] (2005); Alegre, Lapiedra e Chiva (2006); Alegre, Lapiedra e Chiva (2009); Bakar e Ahmad (2010) e Henttonen, Ritala e Juhiainen (2011).

Para Alegre, Lapiedra e Chiva (2006), a eficácia da inovação reflete o seu nível de sucesso. Por outro lado, a eficiência da inovação reflete o esforço feito para alcançar determinado sucesso. Essas duas dimensões de desempenho da inovação de produto são compatíveis com estudos anteriores. A eficácia da inovação versa sobre os resultados econômicos da inovação de produtos, ou seja, sobre o impacto econômico da inovação na empresa, ou, ainda, sobre a importância econômica das saídas do processo de inovação.

A segunda dimensão, Eficiência, se refere a recursos consumidos para atingir esses resultados, isto é, do próprio processo. Destarte, a primeira

Revista de Negócios, v. 21, n. 2, p. 51-66, April, 2016. 
dimensão procura descobrir o que é obtido a partir da inovação (eficácia do resultado de inovação) e a segunda se concentra em como realizar esse processo de inovação (a eficiência do processo). Ambas as dimensões do desempenho inovador são amplamente discutidas na literatura Alegre, Lapiedra e Chiva (2006), Bakar e Ahmad (2010) e Henttonen, Ritala e Juhiainen (2011).

\subsubsection{Desempenho Organizacional}

As informações de Desempenho Organizacional como evolução do faturamento, proporção de vendas nacionais e internacionais são usadas pelas organizações para direcionar seu planejamento, desenvolver estratégias que permitam aproveitar oportunidades, para se defender de possíveis ameaças dos concorrentes e ajudar a compreender como estão sendo usados os recursos materiais, humanos e tecnológicos. Analisar o desempenho organizacional permite disponibilizar ao administrador condições para medir as ações que estão sendo tomado na organização, como sua capacidade de produção, nível de eficácia e eficiência dos processos, resultados de vendas, portanto, existem diversas formas para abordar o desempenho em uma empresa (Neely et al., 1995).

Segundo Miles et al. (1978) a decisão por empreender se apresenta como uma questão essencial colocada para as organizações, sendo as soluções adotadas por elas, definidoras do seu domínio de mercado e das relações de seus produtos com o mercado. Os gestores responsáveis pela administração estratégica se preocupam quanto às consequências das definições dos processos gerenciais, decisões e ações tomadas na organização e seus posteriores reflexos no Desempenho Organizacional (Lumpkin \& Dess, 1996).

O constructo de Desempenho Organizacional, foi medido por meio de quatro itens, sendo dois de desempenho de mercado (fidelidade dos clientes e crescimento das vendas) e dois de desempenho financeiro (rentabilidade e retorno sobre o investimento) baseado no Manual de Oslo da OCDE (2005) e López, Péron e Ordas (2005). Medidas indiretas similares de desempenho organizacional foram utilizados em pesquisa anteriores (López, Péron e Ordas, 2005 e Gunday et al., 2011) quando os dados das demonstrações financeiras estão indisponíveis ou quando eles não permitem comparações precisas entre as empresas. A inovação é um dos instrumentos fundamentais para aumentar a participação de mercado e para fornecer à empresa uma vantagem competitiva (Gunday et al., 2011), tendo um impacto positivo sobre o desempenho das empresas, produzindo uma melhor posição no mercado o que resulta em vantagem competitiva $\mathrm{e}$ desempenho superior.

O modelo desenvolvido para esta pesquisa propõe o desempenho inovador como constructo central, influenciado pela $\mathrm{OE}$ e pelas Fontes de Inovação. Tendo por base essa premissa, pretendese analisar a eficácia na inovação do produto, eficiência no processo de inovação e o Desempenho Organizacional. A seguir, apresentase o modelo teórico (desenho da pesquisa), na figura 1.

Figura 1. Modelo Teórico da Pesquisa

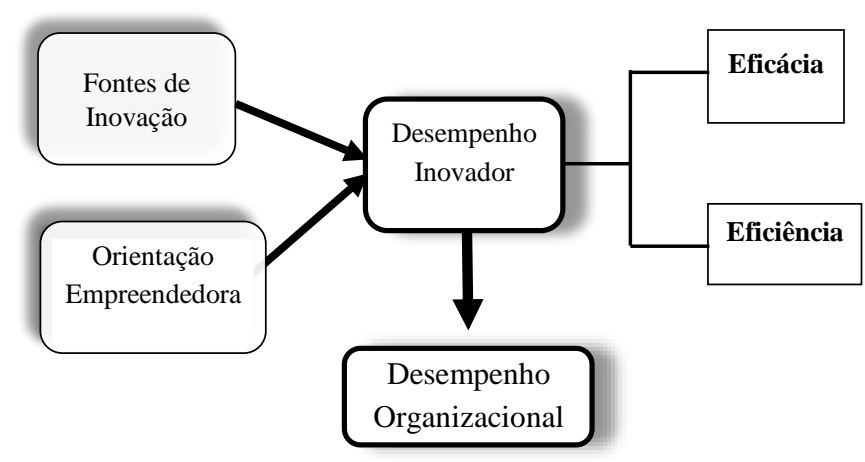

Fonte: Elaboração dos autores.

Conforme a Figura 1 as Fontes de Inovação e a Orientação Empreendedora são tratadas como antecedentes do desempenho Inovador, e este por sua vez está relacionado com o Desempenho Organizacional. O Desempenho Inovador é composto pelas dimensões de Eficiência e Eficácia. As Fontes de Inovação e o Desempenho Organizacional são formados por única dimensão. $\mathrm{Na}$ sequência são apresentados os procedimentos metodológicos.

\section{Metodologia}

Quanto a classificação, a pesquisa foi quantitativa, descritiva, causal e survey com corte transversal. Os dados foram coletados por meio de um questionário fechado com 33 questões.

As assertivas foram construídas por meio de escala Likert de 7 pontos com as seguintes opções: Construto de Fontes de Inovação (1 "Pouco Usado"

Revista de Negócios, v. 21, n. 2, p. 51-66, April, 2016. 
até 7 "Muito Usado"); Constructos de OE (1 "Discordo Totalmente" a 7 "Concordo Totalmente"). Os outros constructos também foram compostos em escala Likert de 7 pontos, mas dentro de um rótulo de concorrência (1 - Muito pior do que os concorrentes a 7 - Muito melhor que os concorrentes) que abrangem os construtos Desempenho Inovador - Eficácia de Inovação de Produtos e Eficiência no Processo de Inovação - e Desempenho Organizacional. O Quadro 1 apresenta os constructos abordados neste trabalho.

O constructo de Fontes de Inovação foi elaborado com base no Manual de Oslo da Organização para Cooperação e Desenvolvimento Econômico, pois possibilita escala de medida adequada dos objetivos das fontes de inovação (OCDE, 2005). No Quadro 1 encontra-se a descrição das assertivas.

Quadro 1. Constructo Fontes de Inovação

\begin{tabular}{|c|c|c|}
\hline Indicador & Assertiva & Autores \\
\hline FINO1 & $\begin{array}{r}\text { Fornecedores de equipamentos, } \\
\text { materiais, componentes ou } \\
\text { software. }\end{array}$ & \\
\cline { 1 - 2 } FINO2 & Clientes ou consumidores. & \\
\cline { 1 - 2 } FINO3 & $\begin{array}{r}\text { Concorrentes e outras empresas } \\
\text { do mesmo setor }\end{array}$ & \multirow{2}{*}{ OCDE } \\
FINO4 & Empresas de consultoria & \\
\cline { 1 - 2 } FINO5 & Feiras, exposições & \\
\cline { 1 - 2 } FINO6 & $\begin{array}{r}\text { Universidades ou outras } \\
\text { instituições de ensino superior }\end{array}$ & \\
\cline { 1 - 2 } FINO7 & $\begin{array}{r}\text { Pesquisas do governo ou } \\
\text { pesquisas privadas sem fins } \\
\text { lucrativos }\end{array}$ & \\
\cline { 1 - 2 } FINO8 & $\begin{array}{r}\text { Outras unidades ou filiais } \\
\text { dentro da empresa. }\end{array}$ & \\
\hline
\end{tabular}

Fonte: Adaptado de OCDE (2005).

O constructo de OE é baseado no estudo de Padrão (2011), tendo este, validado em língua portuguesa a escala usada por Hult, Ketchen e Arrfelt (2007). No Quadro 2 encontra-se a descrição das assertivas.

Quadro 2. Constructo Orientação Empreendedora

\begin{tabular}{|c|c|c|}
\hline Indicador & Assertiva & $\begin{array}{l}\text { Autores } \\
\text { (ano) }\end{array}$ \\
\hline OEMP1 & $\begin{array}{c}\text { Enfatiza pesquisa, } \\
\text { desenvolvimento e } \\
\text { liderança tecnológica. }\end{array}$ & \multirow{3}{*}{$\begin{array}{c}\text { Hult, } \\
\text { Ketchen } \\
\text { Arrfelt } \\
\text { (2007); } \\
\text { Padrão } \\
\text { (2011) }\end{array}$} \\
\hline OEMP2 & $\begin{array}{l}\text { Minha empresa inicia } \\
\text { ações que são imitadas } \\
\text { ou contra-atacadas pelos } \\
\text { nossos concorrentes. }\end{array}$ & \\
\hline OEMP3 & $\begin{array}{l}\text { É rápida para introduzir } \\
\text { novas técnicas de gestão }\end{array}$ & \\
\hline
\end{tabular}

\begin{tabular}{|c|c|}
\hline & $\begin{array}{l}\text { e tecnologias de } \\
\text { operação. }\end{array}$ \\
\hline OEMP4 & $\begin{array}{l}\text { Tem forte propensão } \\
\text { para projetos de alto } \\
\text { risco. }\end{array}$ \\
\hline OEMP5 & $\begin{array}{c}\text { É muito ousada em seus } \\
\text { esforços para maximizar } \\
\text { a chance de aproveitar } \\
\text { oportunidades. }\end{array}$ \\
\hline
\end{tabular}

Fonte: Adaptado de Padrão (2011).

Para a análise do Desempenho Inovador o instrumento de coleta de dados foi adaptado dos estudos de Chiva, Alegre e Lapiedra (2007) e Gomes (2013). O constructo foi dividido em dois blocos, um de eficácia e outro de eficiência. No Quadro 3 encontra-se a descrição das assertivas.

Quadro 3. Constructo Desempenho Inovador - Eficácia e Eficiência

\begin{tabular}{|c|c|c|}
\hline Indicador & Eficácia - Assertivas & Autores \\
\hline IPRD1 & $\begin{array}{c}\text { Substituição de produtos } \\
\text { ultrapassados. }\end{array}$ & \multirow{8}{*}{$\begin{array}{l}\text { OCDE } \\
(2005) ; \\
\text { Chiva, } \\
\text { Alegre e } \\
\text { Lapiedra } \\
(2007)\end{array}$} \\
\hline IPRD2 & $\begin{array}{c}\text { Ampliação da linha de produtos } \\
\text { por meio de produtos } \\
\text { tecnologicamente novos }\end{array}$ & \\
\hline IPRD3 & $\begin{array}{l}\text { Ampliação da linha de produtos } \\
\text { por meio de produtos } \\
\text { tecnologicamente aperfeiçoados. }\end{array}$ & \\
\hline IPRD4 & $\begin{array}{l}\text { Desenvolvimento de produtos } \\
\text { fora do segmento principal } \\
\text { organização (produtos } \\
\text { secundários). }\end{array}$ & \\
\hline IPRD5 & $\begin{array}{l}\text { Desenvolvimento de produtos } \\
\text { que respeitam o meio ambiente } \\
\text { (produtos ecológicos). }\end{array}$ & \\
\hline IPRD6 & $\begin{array}{l}\text { Aumento na participação de } \\
\text { mercado. }\end{array}$ & \\
\hline IPRD7 & $\begin{array}{l}\text { Abertura de novos mercados no } \\
\text { exterior. }\end{array}$ & \\
\hline IPRD8 & $\begin{array}{l}\text { Abertura de novos mercados } \\
\text { nacionais. }\end{array}$ & \\
\hline IPRO1 & $\begin{array}{l}\text { Tempo médio, em semanas, de } \\
\text { desenvolvimento do projeto de } \\
\text { inovação (um projeto de inovação } \\
\text { se refere à criação de um novo } \\
\text { produto ou de um novo } \\
\text { componente). }\end{array}$ & \multirow[t]{4}{*}{$\begin{array}{l}\text { OCDE } \\
(2005) ; \\
\text { Chiva, } \\
\text { Alegre e } \\
\text { Lapiedra } \\
(2007)\end{array}$} \\
\hline IPRO2 & $\begin{array}{l}\text { Tempo médio, em horas, para o } \\
\text { desenvolvimento de projetos de } \\
\text { inovação (horas de trabalho de } \\
\text { todas as pessoas envolvidas). }\end{array}$ & \\
\hline IPRO3 & $\begin{array}{l}\text { Custo médio por projeto de } \\
\text { inovação. }\end{array}$ & \\
\hline IPRO4 & $\begin{array}{l}\text { Grau de satisfação geral com a } \\
\text { eficiência do projeto de inovação. }\end{array}$ & \\
\hline
\end{tabular}

Fonte: Adaptado de Gomes (2013). 
Para o constructo de desempenho Organizacional adotou-se o modelo de Calantone, Cavusgil e Zhao (2002) onde se trabalha com rótulos de comparação com os demais concorrentes. Foram usadas as escalas de 1 a 7 , sendo 1 "muito pior do que os concorrentes", 4 "mesmo nível" e 7 "muito melhor que os concorrentes". No Quadro 4 encontra-se a descrição das assertivas.

Quadro 4. Constructo Desempenho Organizacional

\begin{tabular}{|c|c|c|}
\hline Indicador & Assertiva & $\begin{array}{c}\text { Autores } \\
\text { (ano) }\end{array}$ \\
\cline { 1 - 2 } DEMP1 & Fidelidade dos clientes & $\begin{array}{c}\text { Calantone, } \\
\text { Cavusgil e } \\
\text { Zhao } \\
\text { DEMP2 }\end{array}$ Crescimento das vendas \\
DEMP3 & Rentabilidade & \\
\cline { 1 - 2 } DEMP4 & Retorno sobre o investimento & \\
\hline
\end{tabular}

Fonte: Adaptado de Calantone, Cavusgil e Zhao (2002).

A população da pesquisa foi composta por empresas da indústria de móveis e madeira de Santa Catarina. A amostra foi obtida por meio do contato mantido com a Secretaria de Estado da Fazenda do Estado de Santa Catarina [SEF]. A definição da amostra foi intencional, tendo como base os objetivos da pesquisa. Buscou-se selecionar organizações que podem de alguma forma apresentar características que contribuam com a pesquisa a ser realizada, nomeadamente as que possuem atividades de exportação de móveis.

As empresas do setor de móveis selecionadas para esta pesquisa foram aquelas que possuem o Código Nacional de Atividade Econômica (CNAE) número 3101-2/00: fabricação de móveis com predominância de madeira (envernizados, encerados, esmaltados, laqueados, recobertos com lâminas de material plástico, estofados, para uso residencial e não residencial); fabricação de esqueletos de madeira para móveis; fabricação de móveis embutidos de madeira e acabamento de móveis (envernizamento, esmaltagem, laqueação e serviços similares).

Para classificação do porte de empresa foi usado o critério do Serviço Brasileiro de Apoio às Micro e Pequenas Empresas [SEBRAE] (2005), conforme Tabela 1.

Tabela 1. Critério de classificação das empresas no Brasil pelo SEBRAE

\begin{tabular}{c|c|c}
\hline \multirow{2}{*}{$\begin{array}{c}\text { Porte da } \\
\text { empresa }\end{array}$} & $\begin{array}{c}\mid c \\
\text { Comércio e } \\
\text { Serviços }\end{array}$ & Indústria \\
\cline { 2 - 3 } Microempresa & Até 09 & Até 19 empregados \\
\hline
\end{tabular}

\begin{tabular}{l|c|l}
\hline & empregados & \\
\hline $\begin{array}{l}\text { Organização de } \\
\text { pequeno porte }\end{array}$ & $\begin{array}{c}\text { de } 10 \text { a } 49 \\
\text { empregados }\end{array}$ & $\begin{array}{l}\text { de } 20 \text { a } 99 \\
\text { empregados }\end{array}$ \\
\hline $\begin{array}{l}\text { Organização de } \\
\text { médio porte }\end{array}$ & $\begin{array}{c}\text { de } 50 \text { a } 99 \\
\text { empregados }\end{array}$ & $\begin{array}{l}100 \text { a } 499 \\
\text { empregados }\end{array}$ \\
\hline $\begin{array}{l}\text { Organização de } \\
\text { grande porte }\end{array}$ & $\begin{array}{c}\text { mais de } 99 \\
\text { empregados }\end{array}$ & $\begin{array}{l}\text { mais de 500 } \\
\text { empregados }\end{array}$ \\
\hline
\end{tabular}

Fonte: Adaptado de SEBRAE (2015).

A amostra foi não probabilística, por acessibilidade e conveniência. Neste tipo de amostra, o pesquisador utiliza-se de métodos subjetivos para selecionar os elementos da amostra, pois ela "não é necessariamente feita com o objetivo de ser estatisticamente representativa da população" (Hair et al., 2005, p. 246). Com o intuito de garantir a homogeneidade da amostra, foi incluída uma variável de controle: Tamanho da empresa. No estudo foi utilizada a classificação do [SEBRAE] (2005), que adota o critério do Instituto Brasileiro de Geografia e Estatística [IBGE], classificando as organizações segundo o número de empregados de acordo com o setor de atuação.

A coleta foi realizada em parceria com a SEF. Inicialmente houve um encontro prévio com os gestores da SEF, no sentido de solicitar apoio institucional junto às entidades representativas patronais de classe que estão adstritas ao ramo de fabricação de móveis de madeira de Santa Catarina. As organizações das regiões Oeste, Norte e Serrana do Estado de Santa Catarina por serem as principais regiões produtoras de móveis foram abordadas por meio de telefonemas, sendo parte desta atividade realizada pelo pesquisador e parte pela SEF. Uma vez realizado o telefonema, o e-mail com o link da pesquisa foi enviado para o endereço indicado pela empresa. Foi entregue uma carta de apresentação da pesquisa com o intuito de fomentar a maior participação possível de empresas respondentes informando que os dados obtidos seriam tratados e analisados de forma confidencial e que os resultados seriam divulgados de forma agregada com o intuito a preservar a identidade dos respondentes.

$\mathrm{O}$ instrumento de coleta de dados foi disponibilizado por meio de um link do Google Docs que foi encaminhado por e-mail para as organizações. Durante o progresso dos contatos para coleta de dados verificou-se dificuldade na disponibilização de tempo por parte dos gestores para responderem aos questionários, mesmo com o auxílio da SEF de Santa Catarina entrando em contato com centenas de empresas, houve 63

Revista de Negócios, v. 21, n. 2, p. 51-66, April, 2016. 
respondentes.

\subsection{Perfil da Indústria}

Observa-se uma participação significativa de Santa Catarina no cenário nacional de produção de móveis, possuindo fatias importantes no número de indústrias, trabalhadores empregados, produção, importações e especialmente as exportações, correspondendo a quase $30 \%$ das exportações Brasileiras do setor moveleiro. Os dados do período de 2010 até 2014 que o Inteligência de Mercado [IEMI] (2015) apresenta para Santa Catarina possuem a mesma tendência de crescimento anteriormente demonstrada para o mesmo período no cenário nacional, ou seja, teve crescimento em termos de unidades produtoras, pessoal empregado, valor de produção. No entanto, no que diz respeito aos valores em dólares de exportação houve um recuo de $20,4 \%$ apesar de participação no mercado ter crescido de $25,6 \% \mathrm{em}$ 2012 para 29,3\% em 2014, o que revela questões a serem exploradas para explicarem esse aumento de participação, mas recuo de faturamento em dólares.

A análise dos dados de exportação Santa Catarina esteve em 2014 (e desde 2012) em segundo lugar no ranking nacional, com $29,3 \%$ do mercado, ficando atrás apenas do Rio Grande do Sul com $32,1 \%$. O Paraná, terceiro colocado com $14,1 \%$ fica consideravelmente atrás nessa disputa. Os três estados do Sul são os principais vetores das exportações representando 75,5\% das exportações Brasileiras (IEMI, 2015). As unidades produtoras da indústria de móveis de Santa Catarina são distribuídas por todas as regiões do estado, mas existem dois polos, um em São Bento do Sul e outro em Chapecó que correspondem a $11,5 \%$ e $5,7 \%$ das 2356 unidades produtoras existentes em Santa Catarina em 2014 (IEMI, 2015).

\subsection{Procedimentos de Análise dos Dados}

Os dados obtidos por meio dos questionários foram tabulados em planilha do software Microsoft Excel $^{\odot}$ e posteriormente exportados para o software SPSS (Statistical Package for the Social Sciences) versão 22.0, sendo esta a ferramenta usada para os cálculos e análises estatísticas iniciais relativas a análise descritiva dos dados referente a caracterização das empresas. Após este trabalho inicial, foi analisada a confiabilidade das dimensões pelo Alpha de Cronbach,
Confiabilidade Composta (CC) e a Variância Média Extraída (AVE).

Com esses procedimentos prévios realizados e tendo por base os estudos de Hult, Huerley e Knight (2004), Alegre e Chiva (2005), Alegre e Chiva (2013) que utilizaram a Modelagem de Equações Estruturais (MEE), optou-se por essa técnica pois esta permite a verificação de causa e efeito entre os constructos da pesquisa. A MEE foi realizada com o auxílio do software SmartPLS (Partial least Squares) que possibilita o trabalho de modelos com amostras consideradas pequenas, segundo Wong (2014), com mínimo de 30 a 100 casos (portanto dentro dos números obtidos nesta pesquisa), de acordo com Chin e Newsted (1998). É por meio desta técnica que é possível avaliar a relação entre os constructos e a contribuição de cada item da escala, bem como averiguar o quanto a escala está medindo o conceito, ou seja, sua confiabilidade na estimação das relações entre as variáveis dependentes e independentes (Kline, 2005).

\section{Apresentação e análise dos dados}

\subsection{Caracterização Das Organizações Pesquisadas}

Foi realizada uma verificação de frequência das respostas para possibilitar a identificação do perfil da amostra quanto ao porte e década de fundação. A amostra totalizou 63 respondentes. $\mathrm{O}$ primeiro procedimento realizado foi procurar se existiam outliers nesta base de dados. Com auxílio do software SPSS, foram encontrados 4 respondentes com esta classificação. Desta forma, reduziu-se a base para 59 respondentes. A classificação das empresas quanto ao porte, de acordo com a classificação do SEBRAE (2015) estão descritas na Tabela 2.

Tabela 2. Distribuição da amostra por porte

\begin{tabular}{c|c|c}
\hline Porte & Percentual & Quantidade \\
\hline Microempresa & $28,12 \%$ & 16 \\
\hline $\begin{array}{c}\text { Organização de pequeno } \\
\text { porte }\end{array}$ & $42,18 \%$ & 25 \\
\hline $\begin{array}{c}\text { Organização de médio } \\
\text { porte }\end{array}$ & $28,12 \%$ & 17 \\
\hline $\begin{array}{c}\text { Organização de grande } \\
\text { porte }\end{array}$ & $1,56 \%$ & 1 \\
\hline
\end{tabular}

Fonte: Dados da pesquisa (2016).

Observa-se na Tabela 2 que a maior parte da amostra são de empresas de pequeno porte,

Revista de Negócios, v. 21, n. 2, p. 51-66, April, 2016. 
totalizando 25 com um percentual correspondente a 42,18\%. Houve uma distribuição igualitária entre microempresa e empresas de médio porte com 18 unidades cada uma e com um respectivo percentual de $28,12 \%$. No que diz respeito a empresas de grande porte, somente uma respondeu ao questionário, correspondendo a $1.56 \%$ da amostra. No que diz respeito ao ano de fundação todas participantes informaram este item e a distribuição do quantitativo por década está na Tabela 3.

Tabela 3. Distribuição das empresas por ano de fundação

\begin{tabular}{c|c}
\hline Década & Quantidade \\
\hline 1940 & 1 \\
\hline 1950 & 1 \\
\hline 1960 & 1 \\
\hline 1970 & 5 \\
\hline 1980 & 9 \\
\hline 1990 & 20 \\
\hline 2000 & 7 \\
\hline 2010 & 15 \\
\hline
\end{tabular}

Fonte: Dados da pesquisa (2016).

É possível observar na Tabela 3 que a maioria das empresas que responderam ao questionário se situou com ano de fundação na década de 1990 e 2010, respectivamente.

No que diz respeito aos investimentos com pesquisa em desenvolvimento somente uma empresa respondeu ao quesito, informando que possui um orçamento de 150 mil reais ano destinado a este fim. Com relação ao faturamento, 15 empresas responderam, sendo o valor mínimo de faturamento informado igual a 160 mil reais (ano) e o valor máximo 80 milhões reais (ano).

\subsection{Avaliações Do Modelo De Mensuração}

$\mathrm{Na}$ MEE a primeira fase da avaliação do modelo de mensuração passa pelos seguintes procedimentos: Average Variance Extracted (AVE), Alpha de Cronbach (AC), Composite Reability (CR), Validade discriminante e testes $T$ Student para cada um dos indicadores. Os subitens seguintes irão detalhar cada um destes procedimentos.

\subsubsection{Testes de confiabilidade}

Os testes de confiabilidade realizados nesta pesquisa foram Average Variance Extracted (AVE), Alpha de Cronbach (AC), Composite
Reability (CR). Uma vez montado o modelo foi necessário iniciar com o processo de purificação dos dados. Este procedimento passou pela análise dos valores das cargas fatoriais que o SmartPLS calcula baseado no modelo que foi concebido. $\mathrm{O}$ programa determina as cargas fatoriais existentes entre as variáveis latentes e as variáveis observáveis e a partir de um valor de referência estabelecido de 0,5 sugerido por Chin (1998) procedeu-se a verificação, identificação e retirada dos itens que possuíssem como carga fatorial valores inferiores a 0,5 .

Os indicadores identificados e posteriormente retirados foram: para dimensão Fontes de Inovação: Fontes1, Fontes2, Fontes3, Fontes7. Para o Desempenho Inovador: Eficácia3, Eficácia4, Eficácia6, Eficácia7, Eficiência4. Na dimensão OE, foi retirado somente a variável, OE4. $\mathrm{Na}$ Tabela 4, é possível observar os valores de Alpha de Cronbach (AC), Composite Reability $(C R)$, Average Variance Extracted (AVE) e na coluna número de itens, o valor entre parênteses indica o número de itens que permaneceram no modelo após a exclusão dos itens com carga fatorial inferior a 0,5 .

Tabela 4. Testes de confiabilidade dos constructos da pesquisa

\begin{tabular}{l|c|c|c|c}
\hline \multicolumn{1}{c|}{ Constructo } & $\begin{array}{c}\text { Número } \\
\text { de Itens }\end{array}$ & $\mathbf{A C}$ & $\mathbf{C R}$ & $\mathbf{A V E}$ \\
\hline OE & $5(4)$ & 0,756 & 0,845 & 0,581 \\
\hline $\begin{array}{l}\text { Fontes de } \\
\text { inovação }\end{array}$ & $8(4)$ & 0,758 & 0,847 & 0,583 \\
\hline $\begin{array}{l}\text { Desempenho } \\
\text { Inovador }\end{array}$ & $12(7)$ & 0,842 & 0,881 & 0,515 \\
\hline $\begin{array}{l}\text { Inovação } \\
\text { Produto } \\
\text { (eficácia) }\end{array}$ & $8(4)$ & 0,844 & 0,894 & 0,681 \\
\hline $\begin{array}{l}\text { Inovação } \\
\text { Processo } \\
\text { (eficiência) }\end{array}$ & $4(3)$ & 0,783 & 0,851 & 0,535 \\
\hline $\begin{array}{c}\text { Desempenho } \\
\text { Organizacional }\end{array}$ & $4(4)$ & 0,807 & 0,886 & 0,722 \\
\hline
\end{tabular}

Fonte: Dados da pesquisa (2016).

Ao observar os dados da Tabela 4 é possível evidenciar que no que diz respeito ao critério de AVE todos os constructos situaram-se acima de 0,5 indicando desta forma segundo Heseler, Ringle e Sinkovics (2009) boa confiabilidade nos dados. O constructo que apresentou a maior validade convergente foi Desempenho Organizacional $(0,722)$ o que indica uma relação positiva, confiável para fidelidade de clientes, crescimento

Revista de Negócios, v. 21, n. 2, p. 51-66, April, 2016. 
nas vendas, rentabilidade e retorno sobre investimentos.

Todos os constructos apresentaram boa confiabilidade dos dados uma vez que os valores de Alpha de Cronbach (AC) foram acima de 0,7, em conformidade com a classificação de Hair Jr. et al. (2014). No que diz respeito à Composite Reability $(C R)$, todos os valores ficaram acima de 0,7 o que atesta a boa confiabilidade dos dados segundo. $\mathrm{O}$ constructo Inovação Produto (eficácia) apresentou os maiores valores entre os demais constructos com um valor de $A C$ igual a 0,844 e $C R$ igual a 0,894 . É importante ressaltar que a maioria dos indicadores que permaneceram possuem valores de cargas superiores a 0,7 apresentando patamares elevados, desta forma, reforçando que o modelo proposto teve sua purificação alcançada e valores de qualidade de ajuste (Ringle, Silva \& Bido, 2014).

\subsubsection{Validade Discriminante}

A validade discriminante foi verificada segundo duas formas: primeiro pelo critério de Fornell e Larcker (1981) e segundo pelo modo das cargas cruzadas (Chin, 1998). Este tipo de validade indica como os constructos ou variáveis latentes são independentes um dos outros (Ringle, Silva e Bido, 2014). A Tabela 5 evidencia os resultados obtidos de acordo com Fornell e Larcker (1981).

Tabela 5. Critério de Fornell e Larcker (1981)

\begin{tabular}{|c|c|c|c|c|c|}
\hline 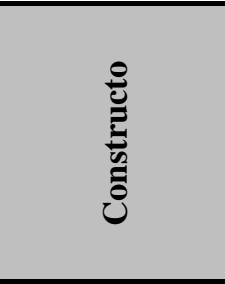 & 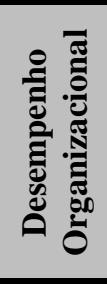 & 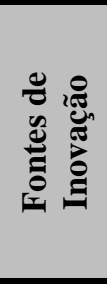 & 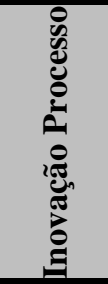 & 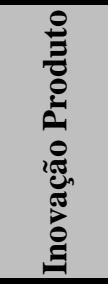 & 됭 \\
\hline $\begin{array}{l}\text { Desempenho } \\
\text { Organizacional }\end{array}$ & 0.825 & & & & \\
\hline $\begin{array}{l}\text { Fontes de } \\
\text { Inovação }\end{array}$ & 0.073 & 0.764 & & & \\
\hline $\begin{array}{l}\text { Inovação } \\
\text { Processo }\end{array}$ & 0.307 & 0.503 & 0.850 & & \\
\hline $\begin{array}{l}\text { Inovação } \\
\text { Produto }\end{array}$ & 0.273 & 0.473 & 0.595 & 0.769 & \\
\hline $\mathrm{OE}$ & 0.078 & 0.582 & 0.389 & 0.538 & 0.762 \\
\hline
\end{tabular}

Fonte: Dados da pesquisa (2016).

Identifica-se (Tabela 5) que os constructos cumprem com o critério de Fornell e Larcker (1981). Neste quesito, de acordo com Ringle, Silva e Bido (2014) são comparadas as raízes quadradas dos valores da AVE dos constructos com as correlações (Pearson) entre os constructos (ou variáveis latentes). As raízes quadradas das AVEs devem ser maiores que as correlações entre os constructos. O valor destacado em cada coluna é maior que os valores abaixo dele para todos os constructos, atestando desta forma o cumprimento do requisito. O segundo critério utilizado, Tabela 6, foi o das cargas cruzadas, conhecido como Cross Loading (Chin, 1998).

Tabela 6. Validade Discriminante segundo o critério Cross Loading

\begin{tabular}{|c|c|c|c|c|c|c|}
\hline \multicolumn{2}{|c|}{ Constructos } & 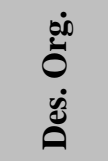 & 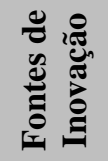 & 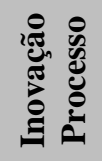 & 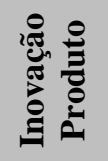 & 됭 \\
\hline \multirow{4}{*}{$\begin{array}{l}\dot{0} \\
\dot{0} \\
\dot{0} \\
\tilde{0}\end{array}$} & Des1 & 0.712 & 0.005 & 0.107 & 0.298 & 0.147 \\
\hline & Des2 & 0.863 & 0.040 & 0.180 & 0.169 & 0.053 \\
\hline & Des3 & 0.847 & 0.072 & 0.268 & 0.182 & -0.016 \\
\hline & Des4 & 0.867 & 0.102 & 0.384 & 0.240 & 0.075 \\
\hline \multirow{4}{*}{ 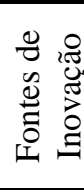 } & Fon4 & -0.117 & 0.809 & 0.379 & 0.333 & 0.451 \\
\hline & Fon5 & 0.075 & 0.646 & 0.320 & 0.463 & 0.537 \\
\hline & Fon6 & 0.238 & 0.790 & 0.379 & 0.263 & 0.380 \\
\hline & Fon8 & 0.045 & 0.798 & 0.445 & 0.356 & 0.387 \\
\hline \multirow{3}{*}{ 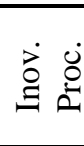 } & Efici.1 & 0.220 & 0.496 & 0.818 & 0.478 & 0.366 \\
\hline & Efici.2 & 0.188 & 0.532 & 0.861 & 0.465 & 0.296 \\
\hline & Efici.3 & 0.365 & 0.269 & 0.870 & 0.569 & 0.330 \\
\hline \multirow{4}{*}{$\begin{array}{l}\dot{D} \\
\stackrel{0}{0} \\
\dot{0} \\
\stackrel{0}{\Xi}\end{array}$} & Eficá1 & 0.187 & 0.369 & 0.430 & 0.824 & 0.529 \\
\hline & Eficá2 & 0.139 & 0.223 & 0.329 & 0.755 & 0.447 \\
\hline & Eficá5 & 0.325 & 0.415 & 0.486 & 0.748 & 0.424 \\
\hline & Eficá8 & 0.181 & 0.429 & 0.564 & 0.747 & 0.262 \\
\hline \multirow{4}{*}{ 띵 } & O_E.1 & -0.022 & 0.416 & 0.254 & 0.372 & 0.630 \\
\hline & O_E.2 & -0.066 & 0.316 & 0.194 & 0.334 & 0.732 \\
\hline & O_E.3 & 0.060 & 0.522 & 0.358 & 0.456 & 0.874 \\
\hline & O_E.5 & 0.208 & 0.481 & 0.342 & 0.453 & 0.792 \\
\hline
\end{tabular}

Fonte: Dados da pesquisa (2016).

A análise das cargas cruzadas descreve mais de uma variável ao mesmo tempo e a frequência da variável é subdividida com os valores das outras variáveis e nela as cargas fatoriais do próprio constructo devem ser maiores que as demais (Chin, 1998) e (Ringle, Silva \& Bido, 2014). Para verificar se existe conformidade com relação a esse critério, percorre-se a tabela de forma horizontal, linha a linha, o constructo na vertical com o constructo na horizontal, observando se o valor daquela célula é superior aos valores das demais células na mesma linha. Ao observar os dados constantes da Tabela 6 é possível verificar que existe, pelo método de carga cruzadas, validade discriminante e por consequência validade dos constructos, uma vez

Revista de Negócios, v. 21, n. 2, p. 51-66, April, 2016. 
que os valores destacados em cinza permitem demonstrar a superioridade dos valores para cada linha da tabela.

Destaca-se que os valores de cada assertiva de todos os constructos são maiores que 1,96 no teste $\mathrm{T}$ de Student e inferiores a $0,05 \mathrm{em}$ todas as assertivas relativas à $\mathrm{P}$-value, desta forma as correlações e os coeficientes de regressão são significantes. Por conseguinte, mantiveram-se todas as assertivas dos constructos. Na sequência, para avalição do modelo estrutural realizou-se cálculo e análise dos valores de $\mathrm{R}^{2}$ (coeficiente de determinação de Pearson), $\mathrm{Q}^{2}$ (relevância ou validade preditiva ou indicador de Stone-Geisser) e do $\mathrm{f}^{2}$ (tamanho do efeito ou indicador de Cohen).Com o coeficiente de determinação de Pearson $\left(\mathrm{R}^{2}\right)$, pode-se avaliar a "porção das variâncias das variáveis endógenas, que são explicados pelo modelo estrutural" (Ringle, Silva \& Bido, 2014, p. 65). Adotando-se como base a sugestão de Cohen (1988), em que $\mathrm{R}^{2}=2 \%$ é classificado como efeito pequeno, $\mathrm{R}^{2}=13 \%$ como efeito médio e $\mathrm{R}^{2}=26 \%$ como efeito grande. $\mathrm{O} \mathrm{Q}^{2}$ avalia quanto o modelo se aproxima do que se esperava dele ou a qualidade da predição do modelo ou acurácia do modelo ajustado " (Ringle, Silva \& Bido, 2014) um modelo perfeito teria $\mathrm{Q}^{2}$ igual a uma unidade o que significa que o modelo reflete a realidade sem erros (Ringle, Silva \& Bido, 2014).

$\mathrm{O} \mathrm{f}^{2}$ é obtido pela inclusão e exclusão dos constructos do modelo (um a um) e avalia-se quanto cada constructo é útil para o ajuste do modelo e também é avaliado pela razão entre a parte explicada pelo modelo e a parte não explicada $\left(\mathrm{f}^{2}=\mathrm{R}^{2} /\left(1-\mathrm{R}^{2}\right)\right.$ (Ringle, Silva \& Bido, 2014). Valores de $\mathrm{f}^{2}$ iguais 0,02 são considerados pequenos, 0,15 são considerados médios e 0,35 grandes (Hair et al., 2014). O software Smart PLS gerou os valores do $\mathrm{R}^{2}$ no modo "PLS

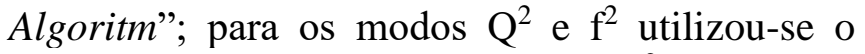
modo "Blindfolding". Os valores de $\mathrm{Q}^{2}$ são obtidos pela leitura da redundância geral do modelo e $\mathrm{f}^{2}$ pela leitura das comunalidades. Na Tabela 7 apresenta-se os valores encontrados.

Tabela 7. Avaliação dos valores de $\mathrm{R}^{2}, \mathrm{Q}^{2}$ e $\mathrm{f}^{2}$

\begin{tabular}{l|c|c|c}
\hline \multicolumn{1}{c|}{ Constructo } & $\mathbf{R}^{\mathbf{2}}$ & $\mathbf{Q}^{\mathbf{2}}$ & $\mathbf{f}^{\mathbf{2}}$ \\
\hline OE & ------ & ------ & 0.289 \\
\hline Fontes de Inovação & ------ & ------ & 0.298 \\
\hline Desempenho Inovador & 0.364 & 0.166 & 0.326 \\
\hline Inovação Processo & 0.777 & 0.553 & 0.421 \\
\hline
\end{tabular}

\begin{tabular}{l|l|l|l}
\hline Inovação Produto & 0.817 & 0.467 & 0.300 \\
\hline Desempenho Organizacional & 0.105 & 0.061 & 0.456 \\
\hline
\end{tabular}

Fonte: Dados da pesquisa (2016).

Os constructos Fontes de Inovação e OE não possuem valor de $\mathrm{R}^{2}$ e $\mathrm{Q}^{2}$ porque antecedem aos outros no Modelo de Equações Estruturais. Ao analisar a Tabela 7 , no que diz respeito ao $R^{2}$ os constructos Desempenho Inovador, Inovação de Processo, Inovação de Produto apresentam efeito grande, e o constructo Desempenho Organizacional efeito aproximado médio. Com relação ao $\mathrm{Q}^{2}$ todos os constructos apresentam valores superiores à zero demonstrando serem uteis para o ajuste do modelo. No que concerne ao $\mathrm{f}^{2}$ os constructos desempenho inovador, fontes de inovação, inovação do produto e OE ficaram num nível médio e próximo da referência grande $(0,35)$, enquanto os constructos Desempenho Organizacional e inovação processo ficaram enquadrados como grandes.

\subsubsection{Valores Teste T de Student e p-value entre os constructos}

$\mathrm{O}$ teste $\mathrm{T}$ de student e p-value entre os constructos procura analisar a relação de relevância entre os constructos. Nesta etapa também se utiliza os valores de T Student indicados como referência que devem ser maior ou igual a 1,96 e p-value menor que 0,05 (Hair et al., 2014). Na Tabela 8 apresentam-se as relações entre os construtos contemplando o Teste $\mathrm{T}$ de Student, $\mathrm{p}$-value e Coeficiente de Caminho $(\Gamma)$.

Tabela 8. Teste T de Student, Coeficiente de Caminho e Pvalue entre os constructos

\begin{tabular}{l|c|c|c}
\multicolumn{1}{c|}{ Constructos } & $\begin{array}{c}\text { Test T de } \\
\text { Student }\end{array}$ & P-value & $\boldsymbol{\Gamma}$ \\
\hline $\begin{array}{l}\text { OE -> Desempenho } \\
\text { Inovador }\end{array}$ & 2.322 & 0.021 & 0.310 \\
\hline $\begin{array}{l}\text { Fontes de Inovação - } \\
\text { > Desempenho } \\
\text { Inovador }\end{array}$ & 2.892 & 0.004 & 0.367 \\
\hline $\begin{array}{l}\text { Desempenho } \\
\text { Inovador -> } \\
\text { Inovação Produto }\end{array}$ & 36.955 & 0.000 & 0.324 \\
\hline $\begin{array}{l}\text { Desempenho } \\
\text { Inovador -> } \\
\text { Inovação Processo }\end{array}$ & 23.991 & 0.000 & 0.881 \\
\hline $\begin{array}{l}\text { Desempenho } \\
\text { Inovador -> } \\
\text { Desempenho } \\
\text { Organizacional }\end{array}$ & 1.959 & 0.050 & 0.904 \\
\hline
\end{tabular}

Fonte: Dados da pesquisa (2016). 
Ao analisar os dados da Tabela 8 destacamse que todas as relações entre constructos, nestes parâmetros, apresentam significância, validando as relações propostas no modelo teórico desta pesquisa. Nesta etapa foi analisado o modelo estrutural completo (figura 2) observando as relações existentes entre os parâmetros identificados nos itens anteriores e também o Coeficiente de Caminho ( $\Gamma$ ) procurando discutir as relações evidenciadas. Para essa atividade foi utilizado o modo PLS Algorithm do SmartPLS.

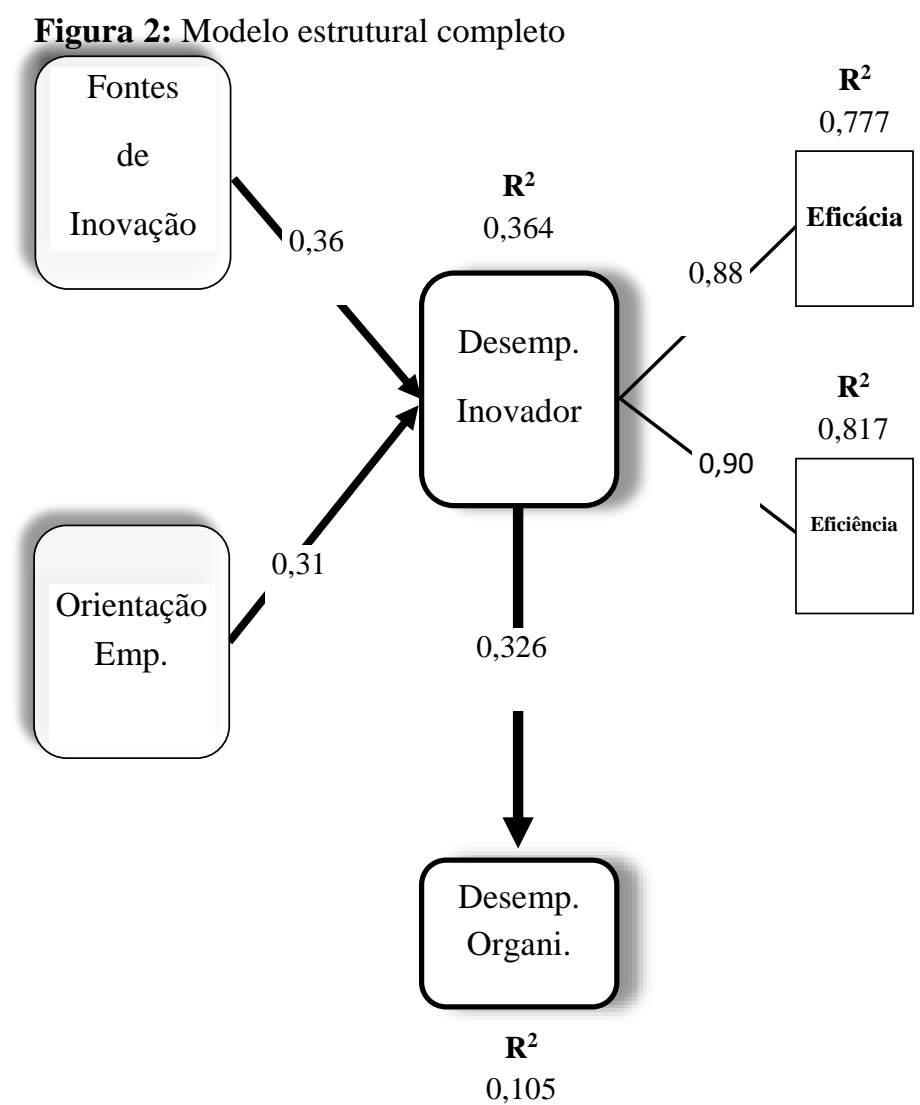

Fonte: Dados da pesquisa (2016).

Conforme pode ser observado na figura $2 \mathrm{e}$ tabela 10 a $\mathrm{OE}$ com coeficiente de caminho $(\Gamma=0.310)$ e $p$-value 0.021 apresentou relação positiva e significativa com o constructo Desempenho Inovador. Levando em consideração a revisão da literatura e a opção por considerar a dimensão $\mathrm{OE}$ abordada de forma multidimensional pelos autores Lumpkin e Dess (1996), Moreno e Casillas (2008) e que os indicadores da OE selecionados nesta pesquisa foram: Assumir riscos, Inovação/Inovatividade e Proatividade e que procura estabelecer uma relação entre os constructos, é relevante discutir como esses indicadores podem estar relacionados com o desempenho inovador.

Neste sentido, destaca-se que a inovatividade segundo Lumpkin e Dess (1996) indica a propensão de uma organização em apropriar ideias novas, fluxos criativos e propiciam por meio destas atividades a geração de novos produtos, processos tecnológicos ou serviços. Por outro lado, é relevante resgatar o conceito de Desempenho Inovador utilizado nesta pesquisa onde o mesmo foi dividido nas dimensões eficácia de inovação de produto e eficiência de processo de inovação propostas por Alegre, Lapiedra e Chiva (2006).

Desta forma, tendo como referência a relação positiva entre OE e Desempenho Inovador é possível sustentar que inovatividade possui um papel positivo na inovação do produto e no processo de inovação. Isso indica que esforços no sentido de gerar e implementar a inovatividade, a assumpção de riscos e a proatividade conduzem a resultados positivos no Desempenho Inovador para as empresas.

Wiklund e Sheperd (2005) argumentam que a $\mathrm{OE}$ pode funcionar como um elemento para superar os problemas existentes em ambientes monótonos, onde existe dificuldade no acesso a recursos financeiros e com poucas oportunidades no mercado de atuação, melhorando os resultados da empresa. Desta forma, a figura do empreendedor pode desempenhar uma função fundamental no encaminhamento de soluções para melhoria dos resultados das pequenas empresas uma vez que nessas organizações a OE, geralmente está centrada no dono da empresa.

A dimensão eficiência, isto é, a dimensão que estuda o processo de inovação que é executado nas fronteiras internas da organização, apresentou maior correlação com o desempenho inovador, com um coeficiente padronizado de $\Gamma=0,904$. Constou-se que as organizações estão preocupadas com o custo e o tempo do projeto despendido com o desenvolvimento da inovação (Alegre, Lapiedra \& Chiva, 2006). Os resultados maiores na dimensão Eficiência, refletem a preocupação com o custo e com o tempo gasto no desenvolvimento dos projetos de inovação.

Pode ser observado na figura 3 que o constructo Fontes de Inovação com (apresentou relação positiva e significativa com o constructo Desempenho Inovador ( $\Gamma=0.367$ e $p$-value 0.004$)$. Primeiro, ao relembrar o conceito vinculado a fontes de inovação é relevante diferenciar as fontes

Revista de Negócios, v. 21, n. 2, p. 51-66, April, 2016. 
internas (normalmente desenvolvidas pelo departamento de pesquisa e desenvolvimento da organização) e externas (instituições públicas de pesquisa nacionais ou internacionais) de mudança (OCDE, 2005).

Segundo a Agência Brasileira de Desenvolvimento Industrial, ABDI (2009) a experiência recente dos principais países produtores de móveis mostra que o acesso aos mercados (variável externa) e a habilidade de reagir (variável interna) rapidamente às mudanças em termos de hábitos e preferências de consumo nesses mercados constituem fatores determinantes do sucesso competitivo das empresas líderes nesse setor.

Para Schoenmakers e Duysters (2006) as rápidas mudanças na tecnologia, muitas vezes, forçam as empresas a depender de conhecimentos e habilidades externas, além dos recursos tecnológicos internos. Muitas empresas estão dependendo mais extensivamente de relações externas para adquirir novos conhecimentos tecnológicos usando estratégias como fornecedores, clientes, concorrentes.

Os órgãos governamentais devem dar maior ênfase à criação de mecanismos ou políticas institucionais eficazes para facilitar a rede de cooperação e estabelecer uma plataforma estável de cooperação. Por exemplo, o apoio numa forma de subsídio financeiro às organizações que conduzem a cooperação em matéria de inovação constitui uma política de incentivos eficaz. (Hemmert, 2004) e (Tsai, 2009). As organizações devem procurar uma maior cooperação com outros parceiros, tais como clientes, universidades, por meio do estabelecimento de redes de cooperação. (Zeng et al. 2010).

Ainda, de acordo com a figura 3 o constructo Desempenho Inovador apresentou relação positiva e significativa $(\Gamma=0.324$ e $p$-value $=0.050)$ com o Desempenho Organizacional. De acordo com Neely et al. (1995) analisar o Desempenho Organizacional permite disponibilizar ao gestor condições para medir as ações que estão sendo tomado na organização, nível de eficácia e eficiência dos processos, resultados de vendas, portanto, existem diversas formas para abordar o desempenho numa empresa. Este resultado está em conformidade com Calantone, Cavusgil e Zhao (2002), os resultados empíricos dos autores indicaram que que a inovação influencia positivamente o desempenho organizacional.
Em uma visão geral, a comparação dos resultados com a literatura nacional e internacional para o teste de validade e consistência dos índices, todos se mostraram adequados aos referenciais existentes, evidenciando que o modelo proposto alcançou o objetivo proposto. Foram encontradas relações positivas da OE e Fontes de Inovação com o desempenho inovador e consequentemente com o Desempenho Organizacional indicando que investir ou reforçar as ações vinculadas a $\mathrm{OE}$ e fontes de inovação propicia retorno no desempenho da empresa.

Esta pesquisa apresentou como novidade a proposição de buscar relacionar os fatores facilitadores da inovação - Fontes de Inovação e $\mathrm{OE}$ - e sua relação com o Desempenho Inovador e Desempenho Organizacional (que ainda não tinha sido realizada) que resultou positiva. O que permite ampliar as relações teóricas existentes pois anteriormente trabalhos relacionavam $\mathrm{OE} e$ Desempenho Organizacional ou OE e Desempenho Inovador. Desta forma, por um lado confirma as relações da literatura existente e permite avançar no sentido da inclusão de um terceiro constructo.

\section{Conclusão}

O presente estudo teve como objetivo avaliar a influência dos fatores facilitadores do desempenho inovador e desempenho organizacional, por meio das Fontes de Inovação e da OE em empresas da indústria de móveis de madeira do estado de Santa Catarina. Constatou-se que as fontes de inovação influenciam positivamente a inovação de produto e também possuem papel relevante como indicador do esforço para obtenção do sucesso em inovação, ou seja, no processo de inovação. É importante considerar que os resultados mostraram a baixa influência das instituições públicas (Universidades, Agências de fomento) na geração da inovação no setor de móveis em Santa Catarina. Como essas Instituições deveriam ter um papel importante no fomento da Inovação, existe uma lacuna provocado por este fato, que dificulta o incremento de inovação no setor.

O Desempenho Inovador foi dividido nas dimensões eficácia de inovação de produto e eficiência de processo. Com base nos resultados da pesquisa é possível sustentar que os indicadores presentes na OE (Assumir riscos, Inovação/Inovatividade e Proatividade) possuem

Revista de Negócios, v. 21, n. 2, p. 51-66, April, 2016. 
um papel positivo na inovação do produto e no processo de inovação. Isso significa que a empresa ao assumir riscos, ser inovadora e ser proativa gera consequências positivas no Desempenho Inovador.

Verificou-se que a inovação de produto (eficácia) e inovação de processo (eficiência), conduz a resultados positivos no Desempenho Organizacional, nomeadamente na fidelidade dos clientes, crescimento das vendas, rentabilidade, retorno sobre $\mathrm{o}$ investimento, que foram os indicadores utilizados nesta pesquisa.

No geral, pode-se concluir que as Fontes de Inovação e a Orientação Empreendedora são importantes facilitadores do Desempenho Inovador e consequentemente do Desempenho Organizacional. Isso ocorre porque a Orientação Empreendedora incorpora as qualidades de proatividade, agressividade e iniciativa que podem impulsionar a organização na direção de novos projetos de inovação. A evidência empírica da pesquisa mostra que tais inter-relações servem para proporcionar vantagem sustentada às organizações e, portanto, são importantes para os gestores que almejam maiores resultados na inovação. Isso se torna mais relevante no setor de Móveis de Madeira que busca inovar constantemente.

\section{Implicações e Pesquisas futuras}

A implicação acadêmica deste trabalho foi a aplicação de uma pesquisa que buscou relacionar Fontes de Inovação, OE, Desempenho Inovador e Desempenho Organizacional, pois não foram encontrados trabalhos considerando estes constructos em simultâneo. Desta forma procurouse analisar os fatores que influenciam o desempenho inovador de produtos e processos e em que dimensão o Desempenho Organizacional é afetado por estes e também em que medida as fontes de inovação e a $\mathrm{OE}$ pode contribuir para o desempenho inovador.

Conforme resultados empíricos, pode-se constatar a relação positiva entre $\mathrm{OE}$, Fontes de Inovação e o Desempenho Inovador. Por sua vez o Desempenho Inovador também apresentou uma relação positiva com os constructos de segunda ordem Inovação de Produto e Inovação de Processo. Finalmente, o Desempenho Inovador apresentou uma relação positiva com o Desempenho Organizacional. Desta forma, o modelo proposto no início da pesquisa foi comprovado.
Além das relações propostas terem se mostrado positivas um aspecto que merece destaque é a questão da centralidade da $\mathrm{OE}$ no empreendedor (dono ou gerente), deste modo, nesta pesquisa o Desempenho Organizacional está intimamente ligado a figura do empreendedor e portanto na presença de Orientação Empreendedora nesta pessoa. Outro dado que reforça essa dependência de resultados empresariais com o empreendedor foi a identificação que os resultados mostraram que a OE teve maior influência no desempenho inovador e consequentemente no Desempenho Organizacional.

Como contribuição gerencial, os dados desta pesquisa, propiciam um conjunto de informações que servem de apoio à tomada de decisões para a área de gestão das empresas de móveis. Por conseguinte, é possível definir com maior propriedade suas metas e necessidades de melhoria de produtos e processos e cogitar ampliar a sua participação em termos de resultados financeiros, no mercado nacional.

No transcorrer da pesquisa algumas limitações foram encontradas. A primeira foi que maioria das empresas não responderam a questões como faturamento, gastos com pesquisa e desenvolvimento, números relativos à atividade de exportação e outras questões. Outra limitação a ser destacada foi a aplicação do questionário somente aos gestores, desta forma, ficaram de fora informações que poderiam ser coletados de chefias intermediárias e dos colaboradores em geral. No entanto, essa opção foi feita porque entende-se que os métodos de trabalho e produção no setor de móveis são bastante rígidos, fechados, com pouca penetração para que essas pessoas que exercem atividades intermediárias possam ser criativas, inovadoras, empreendedoras.

Para pesquisas futuras sugere-se ampliar os esforços no sentido de captar empresas de maior porte, pois entende-se que, devido ao fato destas empresas possuírem um perfil ligado a exportação e com estruturas de gestão mais profissionalizadas, poderão conduzir a resultados que permitam compreender as questões relativas à internacionalização de empresas de móveis. Ainda, pode-se sugerir que esta pesquisa seja aplicada aos Estados do Paraná, Rio Grande do Sul e São Paulo, o que permitirá traçar um perfil mais abrangente da indústria de móveis brasileira uma vez que juntamente com Santa Catarina, estes Estados

Revista de Negócios, v. 21, n. 2, p. 51-66, April, 2016. 
representam a maioria das empresas deste setor. Outro aspecto que pode ser melhor explorado no futuro é procurar identificar quais os motivos da baixa influência das instituições públicas na geração da inovação no setor de móveis em Santa Catarina e porque os mecanismos de inovação não estão sendo acionados por estas organizações.

\section{Referências}

Alegre, J., Lapiedra, R., Chiva, R. (2009). Measuring innovation in long product development cycle industries: an insight in biotechnology. Technology Analysis \& Strategic Management, v. 21, n. 4, pp. 535-546.

Alegre, J., Lapiedra, R., Chiva, R. (2006). A measurement scale for product innovation performance. European Journal of Innovation Management, 9(4), 333-346.

Bakar, A., Ahmad, H. (2010). Assessing the relationship between firm resources and product innovation performance: A resource-based view. Business Process Management Journal, 16(3), 420-435.

Calantone, J., Cavusgil, T., Zhao, Y. (2002). Learning orientation, firm innovation capability, and firm performance. Industrial Marketing Management, 31(6), 515-524.

Chin, W., Newsted, R. (1998). Structural equation modeling analysis with small samples using partials least squares. In: HOYLE, Rick H. Statistical Strategies for Small Sample Research, Sage.

Chiva, R., Alegre, J. (2013). Linking entrepeneurial orientation and firm performance: The role of organizational learning capability and innovation performance. Journal of Small Business Management, 51, 491-507.

Chiva, R., Alegre, J. (2005) Organizational learning and organizational knowledge towards the integration of two approaches. Management learning, 36 (1), 49-68.

Chiva, R., Alegre, J., Lapiedra, R. (2007) Measuring organisational learning capability among the workforce. International Journal of Manpower, 28 (3/4), 224-242.

Damanpour, F. (1991) Organizational Innovation: A meta-analysis of effects of determinants and moderators. Academy of Management Journal. 34(3), 555-590.

Fernández-Mesa, A., Alegre, J. (2015). Enterpreneurial orientation and export intensity: Examining the interplay of organizational learning and innovation. International Business Review, 24(1), 148-156.

Fornell, C., Larcker, D.F., (1981). Evaluating structural equation models with unobservable variables and measurement error. Journal of Marketing Research 18 (1), 39-50.

Freeman, C. (1995) The 'National System of Innovation'in historical perspective. Cambridge Journal of economics, 19(1), 5-24.

George, G., Wood, D. R. Jr, Khan, R. (2001) Networking Strategy of Boards: Implications for Small and Medium-Sized Enterprises. Entrepreneurship and Regional Development, 13, 269-285.

Gomes, G. (2013). Cultura de inovação e sua influência no desempenho em inovação de produtos na indústria têxtil de Santa Catarina. 301

f. Tese (Doutorado) - Curso de Ciências Contábeis e Administração, Universidade Regional de Blumenau, Blumenau, Santa Catarina, Brasil.

Gunday, Gurhan et al. (2011). Effects of innovation types on firm performance. International Journal of production economics, 133(2), 662-676.

Gupta, K., Wilemon, D., Atuahene-Gima, K. (2000). Excelling in r\&d. Research-Technology Management, 43(3), 52-58.

Hair, J., Jr (2014) et al. A primer on partial least squares structural equation modeling (PLS-SEM). Sage Publications.

Hair, J., Jr., Babin, B., Money, A. H., Samouel, P. (2005). Fundamentos e métodos de pesquisa em administração. Porto Alegre: Bookman.

Hemmert, M. (2004). The influence of institutional factors on the technology acquisition performance of high-tech firms: survey results from Germany and Japan. Research Policy,33( 6) 1019-1039.

Henttonen, K., Ritala, P., Jauhiainen, T. (2011) Exploring open search strategies and their perceived impact on innovation performance-

Revista de Negócios, v. 21, n. 2, p. 51-66, April, 2016. 
Empirical evidence. International Journal of Innovation Management, 15(03), 525-541.

Heseler, J., Ringle, C. M., \& Sinkovics, R. R. (2009). The use of partial least squares path modeling in international marketing. Advances in International Marketing, 20, 277-319.

Hult, G. Tomas M., Ketchen, David J., Arrfelt, Mathias. (2007). Strategic supply chain management: Improving performance through a culture of competitiveness and knowledge development. Strategic Management Journal, 28(10), 1035-1052.

Hult, G., Tomas M., Hurley, Robert F., Knight, Gary A. (2004). Innovativeness: Its antecedents and impact on business performance. Industrial Marketing Management, 33(5), 429-438.

Inteligência de Mercado. (2016). Recuperado em 20 de janeiro, 2016, de http://www.iemi.com.br

Inkpen, Andrew C., Pien, W. (2006). An examination of collaboration and knowledge transfer: China-Singapore Suzhou Industrial Park. Journal of Management Studies, 43(4),779811.

Ireland, R. Duane., Reutzel, Christopher R., Webb, Justin W. (2007). Entrepreneurship research in AMJ: what has seen published, and what might the future hold? Academy of Management Journal, 48(4), 556-564.

Ireland, R., Duane., Webb, Justin W., Coombs, E. Joseph. (2005). Theory and Methodology in entrepreneurship research. Research Methodology in Strategy and Management, 2, 111-141.

Kline, R. B. (2005) Principles and practice of structural equation modeling. New York: The Guilford Press.

López, P., Peón, M., José Vazquez Ordás, Camilo. (2005). Organizational learning as a determining factor in business performance. The learning organization, 12(3), 227-245.

Lumpkin, G. T.; Dess, G. (1996). Clarifying the entrepreneurial orientation construct and linking it to performance. The Academic of Management Review, 21(1), 135-172.

Miles, Raymond E. et al. (1978). Organizational strategy, structure, and process. Academy of management review, 3(3), 546-562.
Miller, Danny. (1983). The correlates of entrepreneurship in three types of firms. Management Science, 27(7), 770-791.

Moreno, Ana M., Casillas, José C. (2008) Entrepreneurial orientation and growth of SMEs: A causal model. Entrepreneurship theory and practice, 32(3), 507-528.

Neely, A., Gregory, M., Platts, K. (1995). Performance measurement system design: a literature review and research agenda. International journal of operations \& production management, 15(4), 80-116.

Nelson, R. (1991).Why Do Firms Differ and How Does It Matter? Strategic Management Journal. $12,61-74$.

OCDE - Organização para a Cooperação e o Desenvolvimento. (2005). Manual de Oslo. 3ed. Finep - tradução português.

Padrão, Luis Carlos. Fatores críticos de sucesso no desenvolvimento de produtos de empresas de base tecnológica incubadas. (2011). 197 f. Tese (Doutorado) - Curso de Administração, Fundação Getúlio Vargas, São Paulo, São Paulo, Brasil.

Rauch, A., Wiklund, J., Lumpkin, G., Frese., M. (2009). Entrepreneurial Orientation and business performance: an assessment of past research and sugestions for the future. Entrepreneurial Theory Pratice. 33, 761-787.

Ringle, M., Da Silva, D., Bido., Diógenes de Souza. (2014). Modelagem de equações estruturais com utilização do SmartPLS. REMark, 13(2), 54.

Runyan, R., Droge, C., Swinney, J. (2008). Entrepreneurial Orientation versus Small Business Orientation: What Are Their Relationships to Firm Performance? Journal of Small Business Management, 46, 567-588.

Serviço Brasileiro de apoio as micros e pequenas empresas. (2016). Recuperado em 21 de janeiro, 2016, de http://www.sebrae.com.br.

Schoenmakers, W., Duysters, G. (2006). Learning in strategic technology alliances. Technology analysis \& strategic management, 18(2), 245-264.

Sharma, P., Chrisman, J. (1999). Toward a Reconciliation of the Definitional Issues in the Field of Corporate Entrepreneurship. Entrepreneurship Theory and Practice, 23, 11-27. 
Thoumrungroje, A., Tansuhaj, P. (2005) Entrepreneurial strategic posture, international diversification and firm performance. The Multinational Business Review, 13, 55-73.

Tidd, J., Bessant, J., Pavitt, K. (2005). Managing Innovation: Integrating Technological, Market and Organizational Change. John Wiley \& Sons.

Tsai, Kuen-Hung. (2009). Collaborative Networks And Product Innovation Performance: Toward a contingency perspective. Research policy, 38(5), 765-778.

Wiklund, J., Shepherd, D. (2005). Entrepreneurial orientation and small business performance: a configurational approach. Journal of Business Venturing, 20(1), 71-91.

Wong, Stanley Kam-Sing. (2014). Impacts of environmental turbulence on entrepreneurial orientation and new product success. European Journal of Innovation Management, 17(2), 229249.

Zeng, S. Xie., Xie, Xue M., Tam., Chi Ming. (2010). Relationship between cooperation networks and innovation performance of SMEs. Technovation, 30(3),181-194.

Zott., C. (2003). Dynamic Capabilities And The Emergence Of Intraindustry Differential Firm Performance: Insights from a Simulation Study. Strategic Management Journal, 24, 97-125, 2003. 